\title{
Forward-backward multiplicity correlations in proton-proton col- lisions from several GeV to LHC energies
}

\author{
L. Bravina ${ }^{1, a}$, J. Bleibel ${ }^{2,3}$, R. Emaus ${ }^{1}$, and E. Zabrodin ${ }^{1,4,5}$ \\ ${ }^{1}$ Department of Physics, University of Oslo, PB 1048 Blindern, N-0316 Oslo, Norway \\ ${ }^{2}$ Max-Planck-Institut für Intelligente Systeme, Heisenbergstr. 3, D-70569 Stuttgart, Germany \\ ${ }^{3}$ Institut für Augewandte Physik, Universität Tübingen, Auf der Morgenstelle 10, D-72076 Tübingen, Ger- \\ many \\ ${ }^{4}$ Skobeltsyn Institute of Nuclear Physics, Moscow State University, RU-119991 Moscow, Russia \\ ${ }^{5}$ National Research Nuclear University "MEPhl" (Moscow Engineering Physics Institute), Kashirskoe high- \\ way 31, Moscow, RU-115409, Russia
}

\begin{abstract}
Forward-backward multiplicity correlations in $p p$ collisions at LHC energies are studied with the quark-gluon string model. Comparison with experimental data and with model calculations for lower energies is performed. The model correctly reproduces the linear slope of the correlations, $\left\langle n_{B}\left(n_{F}\right)\right\rangle=a+b_{c o r r} n_{F}$, in the whole energy interval. Positive correlations arise because of mixing of sub-processes with different mean multiplicities. The increase of $b_{\text {corr }}$ with rising collision energy is linked to the increase of the variety of sub-processes going via the soft and hard multi-Pomeron exchanges. For the events with fixed amount of Pomerons the correlation slope $b_{\text {corr }}$ is shown to be essentially zero.
\end{abstract}

\section{Introduction}

The first observation of significant positive correlations between the multiplicity of charged particles emitted in forward and backward hemispheres in $\bar{p} p$ collisions at ISR energies was considered as evidence of long range correlations between the "clusters" of fragmenting system [1]. This phenomenon has attracted a lot of attention, see e.g. [2-8] and references therein. Positive forward-backward (FB) correlations in multiplicity were found in hadronic interactions at energies from several $\mathrm{GeV}$ to $\sqrt{s}=1.8 \mathrm{TeV}$ [9-12]. In contrast, no significant FB correlations were observed in $e^{+} e^{-}$annihilation at energies up to $\sqrt{s}=93 \mathrm{GeV}[13]$ and $133 \mathrm{GeV}[14]$.

It appeared soon that the FB multiplicity correlations possessed the following properties:

- the linear dependence of the averaged multiplicity of charged particles emitted in forward (or backward) hemisphere on the multiplicity of charged particles emitted in the opposite hemisphere, i.e.,

$$
\left\langle n_{B}\left(n_{F}\right)\right\rangle=a+b_{c o r r} n_{F} .
$$

\footnotetext{
ae-mail: larissa.bravina@fys.uio.no
} 


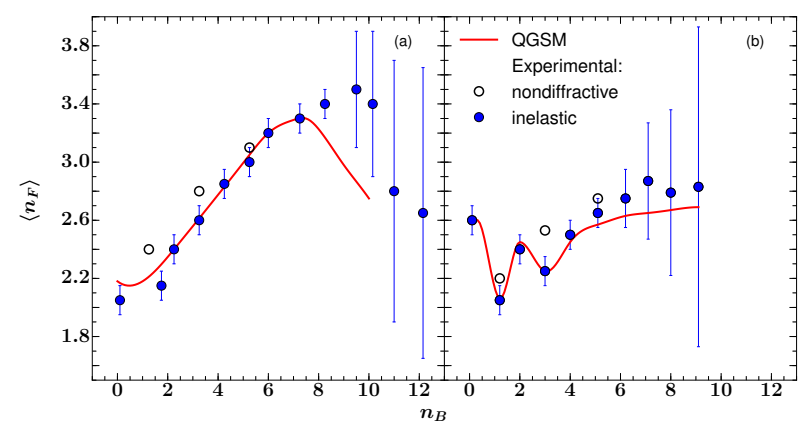

Figure 1. (a): Dependence $\left\langle n_{F}\right\rangle\left(n_{B}\right)$ of charged particles in inelastic (solid circles) and nondiffractive (open circles) $\bar{p} p$ collisions at $32 \mathrm{GeV} / c$. Solid curve denotes the QGSM calculations. (b): The same as (a) but for $p p$ interactions at $32 \mathrm{GeV} / c$.

The slope parameter $b_{\text {corr }}$ is defined as

$$
b_{\text {corr }}=\frac{\left\langle\left(n_{F}-\left\langle n_{F}\right\rangle\right)\left(n_{B}-\left\langle n_{B}\right\rangle\right)\right\rangle}{\left[\left\langle\left(n_{F}-\left\langle n_{F}\right\rangle\right)^{2}\right\rangle\left\langle\left(n_{B}-\left\langle n_{B}\right\rangle\right)^{2}\right\rangle\right]^{1 / 2}},
$$

where $n_{F}$ and $n_{B}$ represent multiplicities of charged particles in forward and backward hemispheres, respectively.

- the correlations reveal positive slope $b_{\text {corr }}$ for particles from the central region $\left|x_{F}\right|<0.1$, whereas for particles from the fragmentation regions $\left|x_{F}\right|>0.1$ the slope is consistent with zero

- the strength of the correlations increases with rising collision energy

- for events with very high particle multiplicity the correlation strength is weakened.

On a phenomenological level these features were explained by mixture of emitting clusters with different mean multiplicity [4]. To provide a linear dependence given by Eq. (1) the clusters should obey the Poisson distribution [8].

We would like to present here our study of forward-backward multiplicity correlations within the quark-gluon string model (QGSM) [15] and its Monte Carlo version [16, 17]. The details of the model can be found in these Proceedings in [18]. QGSM was successfully applied for the description of FB correlations in $p p$ and $\bar{p} p$ collisions at $p_{\text {lab }}=32 \mathrm{GeV} / c$ [10], and recently in $p p$ collisions at LHC energies $900 \mathrm{GeV} \leq \sqrt{s} \leq 13 \mathrm{TeV}$ [19]. Results of the both studies are presented below.

\section{FB correlations in $p p$ and $\bar{p} p$ interactions at intermediate energies}

In [10] the FB multiplicity correlations were studied in both $p p$ and $\bar{p} p$ collisions at $p_{l a b}=32 \mathrm{GeV} / c$. The experiments were carried out on the big bubble chamber "Mirabelle" (ITEP, Serpukhov). Figure 1 shows the $\left\langle n_{F}\right\rangle\left(n_{B}\right)$ dependence of charged particles measured in inelastic and in nondiffractive $p p$ and $\bar{p} p$ interactions. Calculations of QGSM are plotted onto the experimental results as well. The model correctly reproduces the data. One can see that the slope $b_{\text {corr }}$ of the distributions in $\bar{p} p$ collisions is steeper than that in $p p$ collisions. For both reactions, the FB multiplicity correlations seem to be stronger in inelastic collisions compared to non-single diffractive (NSD) ones. 

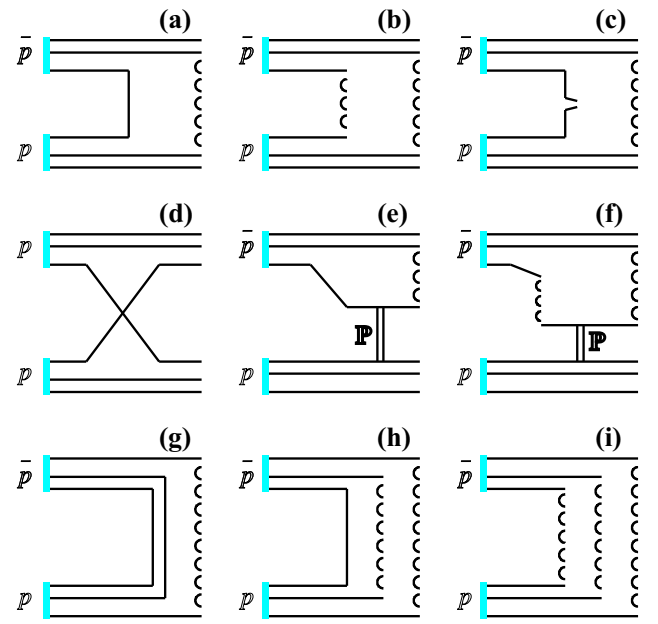

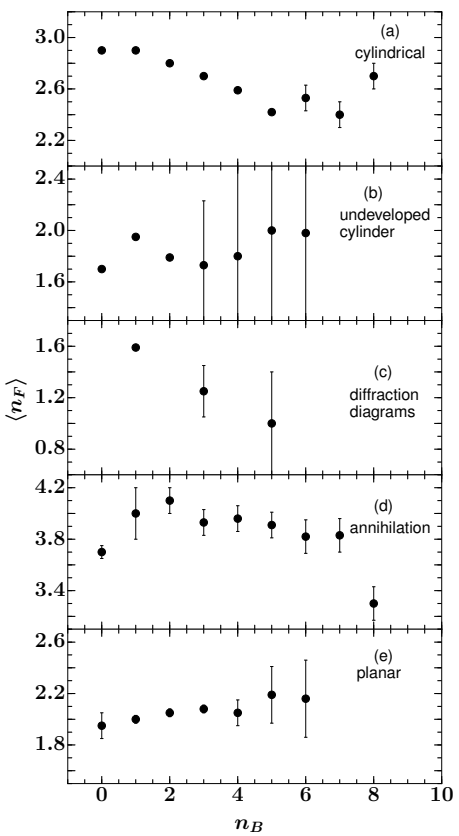

Figure 2. Diagrams taken into account in QGSM in Figure 3. Dependencies $\left\langle n_{F}\right\rangle\left(n_{B}\right)$ for individual mechthe modeling of $\bar{p} p$ and $p p$ interactions at intermediate anisms of $\bar{p} p$ interactions at $32 \mathrm{GeV} / c$ in the QGSM: energies: (a) planar, (b) cylinder, (c) undeveloped cylin- cylinder diagrams (a), undeveloped cylinder diagrams der, (d) binary, (e)-(f) single diffraction with low-mass (b), diffraction diagrams (c), annihilation diagrams (d), and high-mass excitation, (g)-(j) annihilation diagrams. and planar diagrams (e).

Table 1. Slope parameters $b_{c o r r}$ of the forward-backward correlations in inelastic and NSD $p p$ and $\bar{p} p$ collisions at $32 \mathrm{GeV} / c$.

\begin{tabular}{ccc}
\hline \hline Reaction & $p p$ & $\bar{p} p$ \\
\hline non-single diffraction & $0.070 \pm 0.009$ & $0.177 \pm 0.010$ \\
inelastic & $0.098 \pm 0.006$ & $0.192 \pm 0.007$ \\
inelastic, $\left|x_{F}\right|<0.1$ & $0.237 \pm 0.007$ & $0.175 \pm 0.006$ \\
inelastic, $\left|x_{F}\right|>0.1$ & $0.013 \pm 0.006$ & $-0.073 \pm 0.006$ \\
\hline \hline
\end{tabular}

To present this more distinctly, we list the slope parameters in Table 1 for inelastic and NSD events. The data were also extracted for central $\left(\left|x_{F}\right|<0.1\right)$ and for non-central $\left(\left|x_{F}\right|>0.1\right)$ regions in inelastic collisions. The positive FB correlations are observed for central region $\left|x_{F}\right|<0.1$ only, whereas for the regions with $\left|x_{F}\right|>0.1$ there are practically no correlations. 

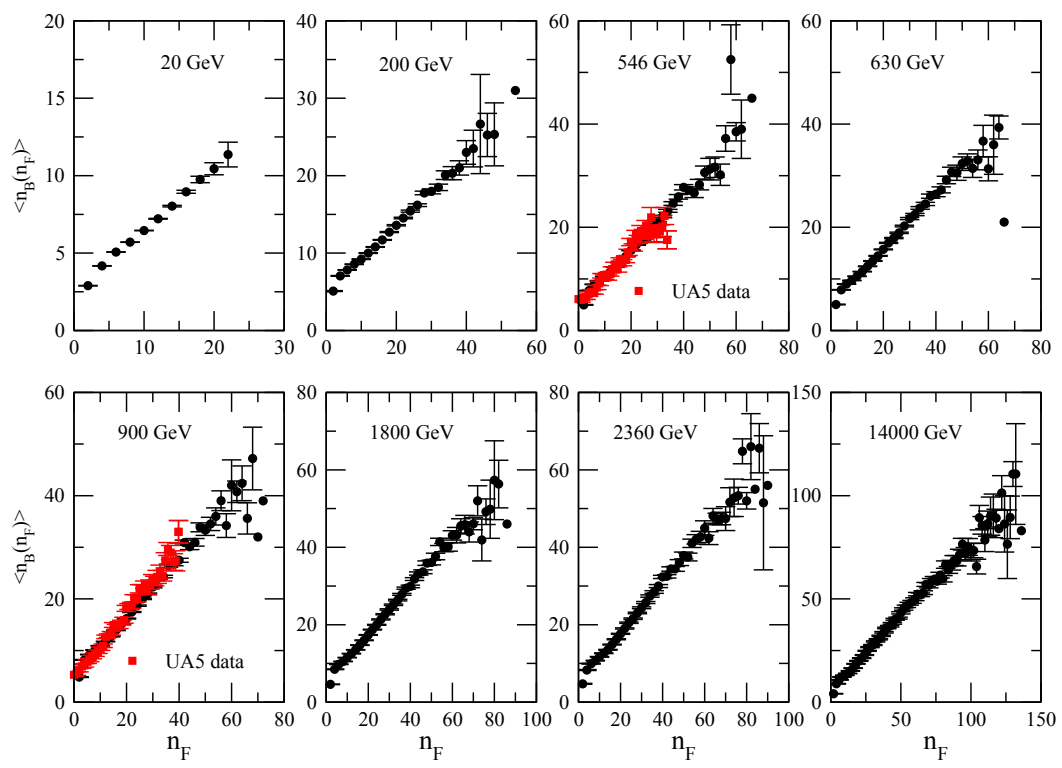

Figure 4. Dependencies $\left\langle n_{B}\right\rangle\left(n_{F}\right)$ for rapidity interval $0 \leq \eta \leq 4$ in NSD $p p$ collisions at $20 \mathrm{GeV} \leq \sqrt{s} \leq 14 \mathrm{TeV}$. Data for $546 \mathrm{GeV}$ and $900 \mathrm{GeV}$ (from [3]) are shown by full squares.

For better understanding of the reason why the FB correlations in $\bar{p} p$ collisions are stronger at this energy than the FB correlations in $p p$ collisions, we plot in Fig. 2 the set of diagrams employed in QGSM for treatment of both types of collisions at intermediate energies. Obviously, the variety of sub-processes in $\bar{p} p$ interactions is richer compared to that in $p p$ ones, because, e.g., the planar diagrams (a) and annihilation diagrams (g)-(i) are absent in $p p$ processes. Since more sub-processes with different mean multiplicities contribute to particle production in $\bar{p} p$ collisions, the correlation slope is steeper, i.e. $b_{c o r r}^{\bar{p} p}>b_{c o r r}^{p p}$. Similarly, because of the lack of single diffraction diagrams the correlation strength in non-single diffractive (NSD) processes is smaller compared to that in inelastic ones. Figure 3 displays the FB correlations for different sub-processes shown in Fig. 2, namely, cylinder diagram, undeveloped cylinder, single diffraction, $\bar{p} p$ annihilation and planar diagram. Only the planar sub-processes demonstrate weak positive FB correlations, whereas the correlation slopes for cylinder and diffractive diagrams are slightly negative.

The cross section of annihilation process drops rapidly with rising energy of the collisions. Therefore, the slopes of FB multiplicity correlations in $p p$ and $\bar{p} p$ interactions become similar after some collision energy threshold. The strength of the correlations continues to increase, as shown in Fig. 4. Here QGSM calculations of FB correlations in $p p$ interactions are displayed for energies ranging from $\sqrt{s}=20 \mathrm{GeV}$ to $\sqrt{s}=14 \mathrm{TeV}$. Comparison with the UA5 data on $\bar{p} p$ collisions at $\sqrt{s}=546 \mathrm{GeV}$ and $900 \mathrm{GeV}[3,11]$ demonstrates a good agreement between the model calculations and the experimental data. For all reactions the slope of the FB correlations remains almost linear. We have mentioned already the vanishing of annihilation diagrams, as well as other so-called pre-asymptotic diagrams, with the increase of collision energy. However, the only reason of enlargement of the correlation strength $b_{\text {corr }}$ in the model is the rise of the variety of different sub-processes with different mean multiplic- 

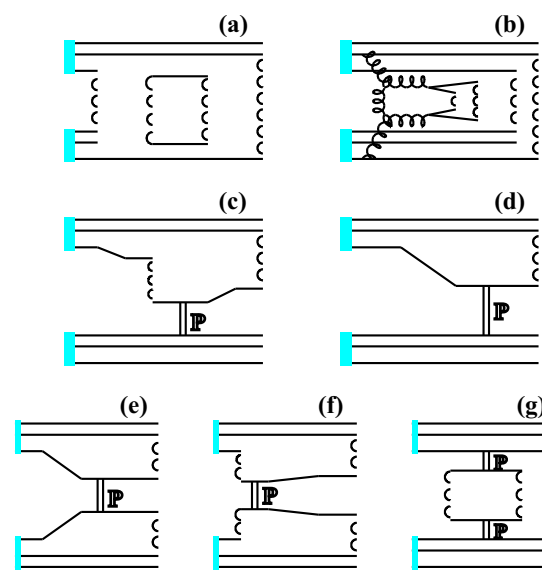

(b)

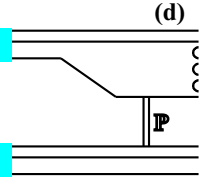

(g)
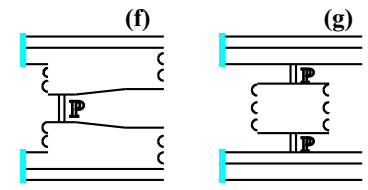

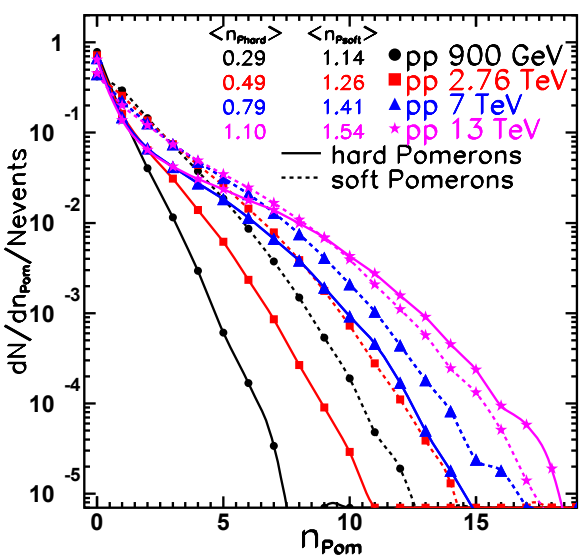

Figure 5. Diagrams taken into account in QGSM in Figure 6. Average numbers of soft (dotted curves) and the modeling of $p p$ interactions at ultrarelativistic en- hard (solid curves) Pomerons per event in $p p$ collisions ergies: (a) multi-Pomeron exchange, (b) (semi)hard at $\sqrt{s}=0.9,2.76,7$, and $13 \mathrm{TeV}$, respectively. gluon-gluon interaction and soft Pomeron exchange, (c)-(d) single diffraction with high-mass and low-mass excitation, (e)-(f) double diffraction with low-mass and high-mass excitation, $(\mathrm{g})$ central diffraction.

ity. These sub-processes and their role in formation of FB multiplicity correlations at ultra-relativistic energies are discussed in 3 .

\section{FB correlations at LHC energies}

The set of diagrams describing the $p p$ interactions at ultra-relativistic energies is shown in Fig.5. The first two diagrams represent (a) the soft (multi)Pomeron exchanges and (b) processes going via the formation of hard Pomerons. Other diagrams deal with the processes of single diffraction, (c) and (d), and double diffraction, (e)-(g). The main contribution to particle multiplicity comes from the processes with soft and hard Pomeron exchanges. Their numbers increase with rising collision energy, as it can be seen in Fig. 6. This figure displays the relative amounts of soft and hard Pomerons in a single $p p$-event at energies $\sqrt{s}=900 \mathrm{GeV}, 2.76 \mathrm{TeV}, 7 \mathrm{TeV}$ and $13 \mathrm{TeV}$, respectively. At $\sqrt{s}=900 \mathrm{GeV}$ the number of soft Pomerons exceeds the number of hard Pomerons by factor 4 , whereas at $\sqrt{s}=13 \mathrm{TeV}$ their ratio drops to 1.4. As it follows from Fig. 6, the maximum number of both soft and hard Pomerons in a single event gradually increases with collision energy. The variety of sub-processes containing all possible combinations of soft and hard Pomerons becomes more extensive. Since these processes have different mean multiplicities of produced hadrons, the strength of the FB correlations measured for the whole sample of events increases, as shown in Fig. 7. One can see that the FB correlations are not very strong at low multiplicity area, $n^{c h} \leq 15$. The slopes of the distributions are also becoming less steep after a certain multiplicity threshold. 


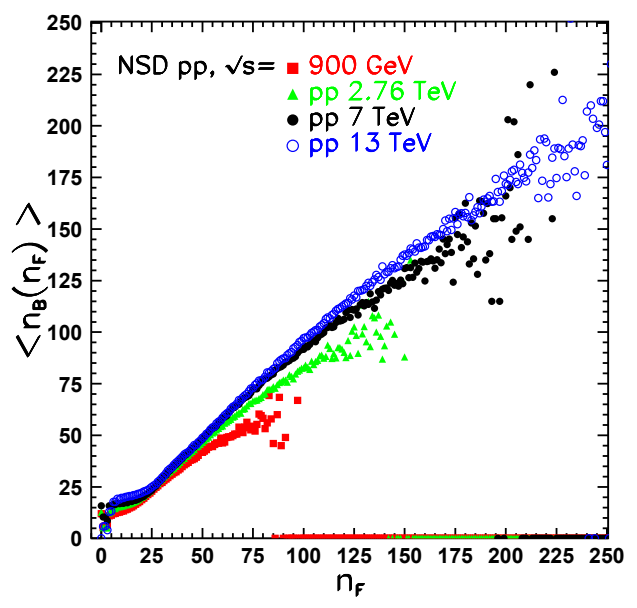

Figure 7. Forward-backward multiplicity correlations in NSD $p p$ collisions at $\sqrt{s}=0.9,2.76,7$, and $13 \mathrm{TeV}$.

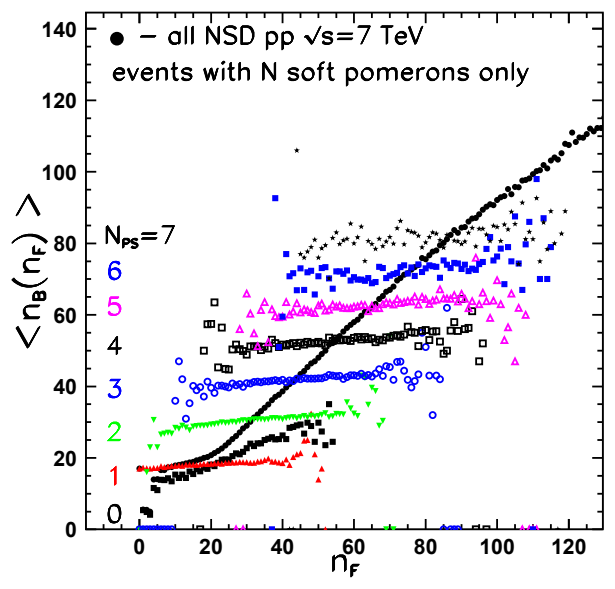

Figure 8. Dependence of average multiplicity $\left\langle n_{B}\right\rangle$ on $n_{F}$ (solid circles) in NSD $p p$ collisions at $\sqrt{s}=7 \mathrm{TeV}$ in QGSM. Curves indicate $\left\langle n_{B}\left(n_{F}\right)\right\rangle$ distributions for subevents with only soft Pomerons, $N_{S_{-} P o m}=0,1, \ldots, 7$.

Figure 8 displays the $\left\langle n_{B}\right\rangle\left(n_{F}\right)$ dependencies calculated in QGSM for $p p$ events at $\sqrt{s}=7 \mathrm{TeV}$ which proceed only via the soft Pomeron exchanges. The FB correlations for the sub-processes with fixed amount of soft Pomerons, varying from 1 to 7, are also plotted in Fig. 8. It is worth noting that FB correlations within each of the selected sample of events have zero slope, although the charged particle multiplicity measured on event-by-event basis changes from few hadrons up to one hundred. Not all topologies contribute to event with very small and very big multiplicity. This circumstance leads to reduction of the correlation strength in both multiplicity intervals.

At LHC energies the FB correlations in $p p$ interactions were studied by the ALICE [21] and ATLAS [22] Collaborations. The analysis is performed in terms of gaps in pseudorapidity, $\eta_{\text {gap }}$, between the hadrons in forward and backward hemispheres, and widths of pseudorapidity bins, $\delta \eta$. Figure 9 presents the comparison of model calculations of the $b_{\text {corr }}$ as a function of $\delta \eta$ at zero rapidity gap with the ALICE data for $\sqrt{s}=900 \mathrm{GeV}, 2.76 \mathrm{TeV}$ and $7 \mathrm{TeV}$. The slope $b_{\text {corr }}$ increases with broadening of $\delta \eta$ for all three energies. Note, that the strength of FB correlations drops with rising midrapidity gap [19].

ALICE Collaboration has also studied the FB correlations between different azimuthal sectors. Parameters of the study are as follows. The azimuthal angle of the sectors is $\varphi=\pi / 4$ and the width of the bin is $\delta \eta=0.2$. The data obtained for $p p$ interactions at $900 \mathrm{GeV}$ and $7 \mathrm{TeV}$ are shown in Fig. 10 in comparison with the QGSM calculations. The correlations at $\sqrt{s}=7 \mathrm{TeV}$ are twice stronger than that at $900 \mathrm{GeV}$; other characteristics are pretty similar.

\section{Conclusions}

We apply the quark-gluon string model, based on Reggeon Field Theory, for the description of forward-backward multiplicity correlations in proton-proton collisions at ultrarelativistic energies. It 


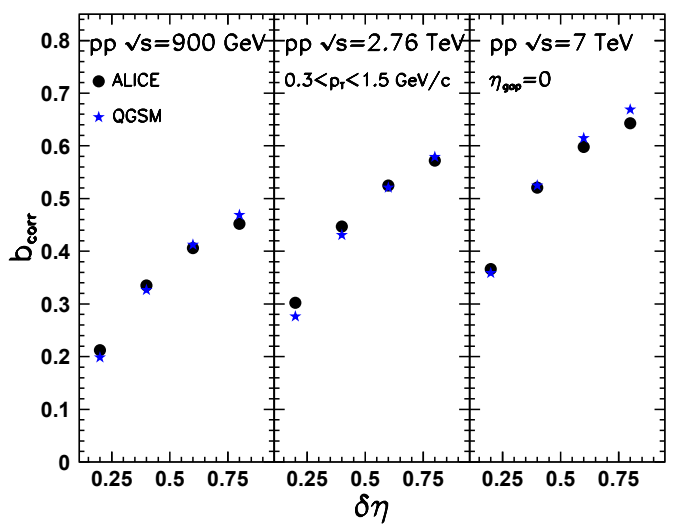

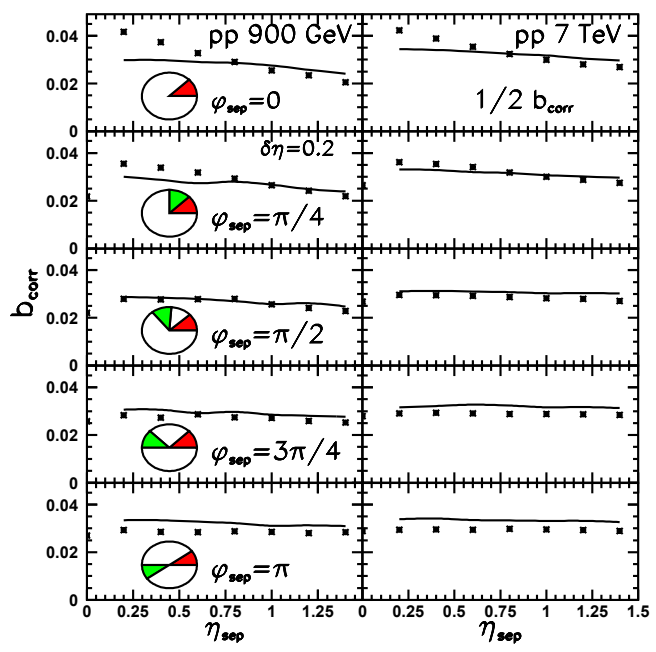

Figure 9. FB correlation parameter $b_{\text {corr }}$ at $\eta_{\text {gap }}=0$ Figure 10. FB correlation parameter $b_{\text {corr }}$ for azin four rapidity bins: $\delta \eta=0.2,0.4,0.6,0.8$ in $p p$ colli- imuthally separated sectors of $\varphi=\pi / 4$ as a function sions at $\sqrt{s}=900 \mathrm{GeV}, 2.76 \mathrm{TeV}, 7 \mathrm{TeV}$ and $13 \mathrm{TeV}$. of midrapidity gap at fixed $\delta \eta=0.2$ in $p p$ collisions Stars and circles denote the model calculations and the at $\sqrt{s}=900 \mathrm{GeV}$ (left) and $7 \mathrm{TeV}$ (right). Results for ALICE data from [21], respectively. note the model calculations and the data from [21], respectively.

is shown that positive FB correlations arise in QGSM because of addition of different sub-processes with different mean multiplicities. For the individual sub-processes, their $\left\langle n_{B}\right\rangle\left(n_{F}\right)$ distributions are remarkably flat, although the event-by-event multiplicity can vary from few particles up to more than one hundred.

At c.m. energies about $10 \mathrm{GeV}$ the number of diagrams describing $\bar{p} p$ collisions is larger than that for $p p$ interactions. Therefore, FB correlations are stronger in $\bar{p} p$ case. At c.m. energies higher than $100 \mathrm{GeV}$ the sets of diagrams for $\bar{p} p$ and $p p$ collisions are similar. The further rise of the correlation strength occurs due to increasing number of soft and hard Pomerons allowed for a single event.

The correlation dependence is linear, $\left\langle n_{B}\right\rangle\left(n_{F}\right)=a+b_{c o r r} n_{F}$, for $\bar{p} p$ and $p p$ collisions at all energies in question. However, for low and for very high multiplicities $n_{F}$ the slopes $b_{c o r r}$ are not so steep. Finally, the FB correlations take place mainly in the region $\left|x_{F}\right|<0.1$, whereas for $\left|x_{F}\right|>0.1$ the correlations are almost absent.

\section{Acknowledgements}

Fruitful discussions with M. Bleicher, L. Csernai and V. Vechernin are gratefully acknowledged. L.B. acknowledges financial support of the Alexander von Humboldt Foundation. J.B. thanks the German Research Foundation (DFG) for the financial support through the Project BL 1286/2-1.

\section{References}

[1] S. Uhlig, I. Derado, R. Meinke, H. Preissner, Nucl. Phys. B 132, 15 (1978) 
[2] K. Alpgaard et al., UA5 Collab., Phys. Lett. B 123, 361(1983)

[3] G.G. Alner et al., UA5 Collab., Phys. Rep. 154, 247 (1987)

[4] T.T. Chou, C.N. Yang, Phys. Lett. B 135, 175 (1984)

[5] A. Capella, A. Krzywicki, Phys. Rev. D 18, 4120 (1978)

[6] A. Capella, J.Tran Thanh Van, Z. Phys. C 18, 85 (1983)

[7] J. Dias de Deus, Phys. Lett. B 100, 177 (1981)

[8] M.A. Braun, C. Pajares, V.V. Vechernin, Phys. Lett. B 493, 54 (2000)

[9] E.G. Boos et al., Phys. Scripta 15, 305 (1977)

[10] L.V. Bravina et al., Sov. J. Nucl. Phys. 50, 245 (1989)

[11] R.E. Ansorge et al., UA5 Collab., Z. Phys. C 37, 191 (1988)

[12] T. Alexopoulos et al., E735 Collab., Phys. Lett. B 353, 155 (1995)

[13] W. Braunschweig et al., TASSO Collab., Z. Phys. C 45, 193 (1989)

[14] R. Akers et al., OPAL Collab., Phys. Lett. B 320, 417 (1994)

[15] A.B. Kaidalov, Phys. Lett. B 116, 459 (1982);

A.B. Kaidalov and K.A. Ter-Martirosyan, Phys. Lett. B 117, 247 (1982)

[16] N.S. Amelin, L.V. Bravina, Sov. J. Nucl. Phys. 51, 133 (1990)

[17] J. Bleibel, L.V. Bravina, E.E. Zabrodin, Phys. Rev. D 93, 114012 (2016)

[18] E.E. Zabrodin et al., these Proceedings.

[19] L.V. Bravina, J. Bleibel, E.E. Zabrodin, Phys. Lett. B (submitted).

[20] G. J. Alner et al., UA5 Collab., Phys. Rep. 154, 247 (1987)

[21] J. Adam et al., ALICE Collab., J. High Energy Phys. JHEP05, 097 (2015)

[22] G. Aad et al., ATLAS Collab., J. High Energy Phys. JHEP07, 019 (2012) 